\title{
Ultramikro-Methode zur Bestimmung des Stickstoffes in biologischem Material')
}

\author{
Von L. Strauch \\ Aus dem .Max-Planck-Institut für Eiveiß- und Lederforscbung, München (Direktor: Prof. Dr. W. Graßmann)
}

(Eingegangen am 24. Februar 1965)

\begin{abstract}
Es wird eine einfache kolorimetrische Ultramikro-Methode zur Bestimmung von Kjeldahl-Stickstoff im biologischen Material beschrieben. Die Proben werden in Reagenzgläsern mit Hilfe eines Aufschlußgerätes, das näher erläutert wird, bei $320^{\circ}$ aufgeschlossen, mit Wasser verdünnt, direkt nesslerisiert und kolorimetrisch ausgewertet. Die Methode kann im Bereich von $1 \mu \mathrm{g}$ bis $1700 \mu \mathrm{g}$ Stickstoff angewandt werden und eignet sich gut zur routinemäßigen Stickstoffanalyse.
\end{abstract}

A simple colorimetric ultramicro method for the determination of Kjeldahl nitrogen is presented. The samples are digested in test tubes at $320^{\circ}$ by means of a digestion apparatus described in the text. The acid digest is diluted, directly nesslerized and read in a spectrophotometer. The method can be used for nitrogen levels from $1 \mu \mathrm{g}$ to $1700 \mu \mathrm{g}$ and has proved to be satisfactory and useful for the routine analyses of nitrogen.

In der letzten Zeit wurden zahlreiche Modifikationen der klassischen Kjeldahlmethode zur Bestimmung von Mikro- und Ultramikromengen von Stickstoff im biologischen Material entwickelt. Verfahren, die mit der Destillation (1-9) oder der Diffusion (10-13) des freigewordenen Ammoniaks arbeiten, verlangen besondere Apparaturen, sind mit einer relativ hohen Fehlergrenze und einem erheblichen experimentellen Aufwand verbunden. Aus diesem Grunde greift man bei täglicher Routinearbeit immer mehr nach direkten kolorimetrischen Methoden. Sie ermöglichen unter Einsparung von Zeit und Arbeitskräften eine größere Anzahl von Analysen zu bewältigen. Bei diesen Verfahren wird das entstandene Ammoniak mittels einer Farbreaktion mit Phenol-Hypochlorit-Reagens (14), mit Ninhydrin $(12,13,15-17)$ und in den meisten Fällen mit Nesslerreagens (18-25) unmittelbar in der Aufschlußlösung kolorimetrisch bestimmt.

Die hier beschriebene Methode weist gegenüber den oben erwähnten Verfahren mehrere Vorteile auf. Der ganze Analysengang wird in Reagenzgläsern ohne jegliches Umgießen der Probenlösungen ausgeführt. Die Vorbereitung des Aufschlußreagens wurde weiter vereinfacht. Als wesentlicher Vorteil unserer Methode kann die Einführung eines von uns entwickelten Aufschlußgerätes betrachtet werden. Dieses Gerät ermöglicht eine gute und schnelle Steuerung des Aufschlußvorganges. Die Aufschlußzeit, die bei einigen Methoden bis zu 12 Stdn. dauert (28), konnte auf diese Weise auf etwa 30-60 Min. vẹkürzt werden. Die aufgeschlossenen Proben werden dann verdünnt, in Anlehnung an die Methode von C. A. LANG (22) direkt nesslerisiert und kolorimetrisch ausgewertet.

Die Methode wird von uns und einigen anderren Laboratorien seit 2 Jahren mit Erfolg zur Stickstoffbestimmung in Körperflüssigkeiten, Proteinen, Geweben, Hautextrakten, Gewebskulturen, sowie in zahlreichen aus Enzymreinigungen durch Chromatographie, Gelfiltration und kontinuierliche Elektrophorese gewonnenen Fraktionen angewandt.

1) Die Arbeit wurde durch ein Stipendium der Helen Hay Wbitney Foundation, New York (F-94) unterstützt.

\section{Methodik}

\section{Reagentien}

Es wurden Chemikalien des Reinheitsgrades pro analysi sowie deionisiertes Wasser mit einer Leitfähigkeit unter $0,1 \mu \mathrm{S}$ verwendet.

\section{Aufschlußreagenz}

$2 \mathrm{~g}$ Selen werden in $250 \mathrm{~m} l$ konzentrierter Schwefelsäure so lange erhitzt, bis die Lösung klar und farblos geworden ist. Nach dem Erkalten werden tropfenweise und unter Kühlung $250 \mathrm{ml}$ einer gesättigten Kaliumsulfatlösung zugegeben.

\section{Nesslerreagenz}

Vorbereitung nach КосH und McMeEKIN (26). Die Lösung wird im Dunkeln aufbewahrt und ist über $1 \mathrm{Jahr}$ haltbar.

\section{Stickstoff-Standardlösungen}

$1,179 \mathrm{~g}$ Ammoniumsulfat (im Vakuum über Phosphorpentoxyd getrocknet) wurden in $250 \mathrm{ml} 0,2 n$ Schwefelsäure gelöst. 65,2 mg L-Lysinmonohydrochlorid wurden in $100 \mathrm{~m} /$ Wasser gelöst. Beide Lösungen enthalten $100 \mu \mathrm{g}$ Stickstoff $/ \mathrm{m} /$. Von diesen Lösungen wird eine Verdünnungsreihe entsprechend $5-100 \mu \mathrm{g} \mathrm{N}$ parallel zur Untersuchungsprobe angesetzt.

\section{Apparaturen \\ Aufscblußgerät ${ }^{2}$ )}

Der Aufschluß erfolgt in einem elektrisch beheizten Aluminiumblock, der mit einem stufenlos einstellbaren Temperaturregler ausgestattet ist (Abb. 1). Die Bohrungen im Block dienen zur Aufnahme von Reagenzgläsern $16 \times 160 \mathrm{~mm}$. Die Beheizung erfolgt durch eine flache im unteren Teil des Blockes angebrachte Heizspirale $(220 \mathrm{~V}, 700 \mathrm{~W})$.

\section{Glasgeräte}

Für die Bestimmung sind nur Reagenzgläser und Pipetten notwendig.

\section{Photometer}

Die optischen Messungen wurden in der vorliegenden Arbeit mit einem Beckman DU Spektrophotometer ausgeführt.

\section{Ausführung}

\section{Anfschlus}

Jedes Reagenzglas wird mit einer Probe, die $5 \mathrm{mg}$ Trockengetwicht oder $1,0 \mathrm{ml}$ Volumen nicht übersteigen soll, beschickt und je $0,2 \mathrm{~m} l$ Aufschlußreagens zugesetzt. Die Reagenzgläser mit den

2) Das Aufschlußgerät wird unter der Bezeichnung „Thermoblock " von der $\mathrm{Fa}$. Wissenschaftlich-Technische Werkstätten G. m. b. H., 8120 Weilheim/Obb., Trifthofs:raße hergestellt. 


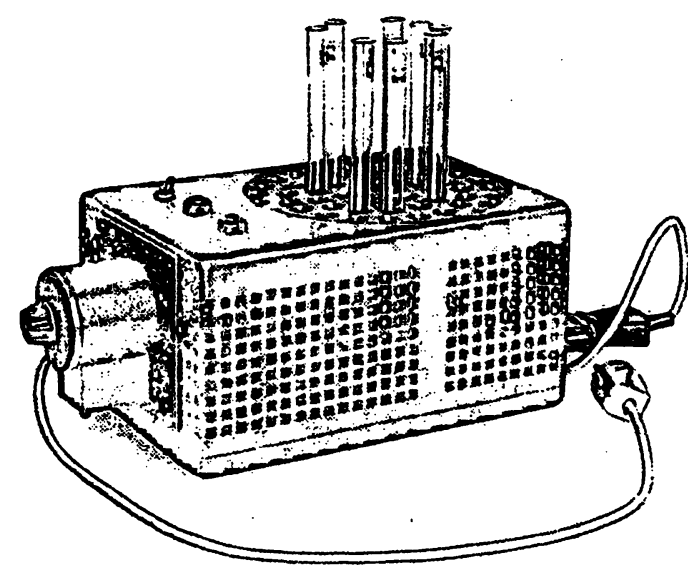

Abb. 1

Das Aufschlußgerät mit eingesetzten Reagenzgläsern

Proben sowie Reagenzgläser mit analog behandelten Standardlösungen werden in die Bohrungen des auf $100^{\circ}$ vorgeheizten Aufschlußgerätes eingesetzt. Der Temperaturreg!er wird auf $320^{\circ}$ eingestellt. Nach etwa 20 bis $30 \mathrm{Min}$. ist die gewünschte Temperatur erreicht. Danach wird der Aufschluß weitere 30 Min. fortgesetzt. Die Reagenzgläser werden dann aus dem Aufschlußgerät entfernt und bis zur Abkühlung auf Zimmertemperatur stehengelassen. Das Volumen der klaren Lösung in jedem Reagenzglas beträgt nun $0,1 \mathrm{~m} /$.

\section{Nesslerisierung}

Verdünnungsmethode: Die sauren Proben werden mit Wasser auf $10,0 \mathrm{~m} /$ aufgefüllt und zur Bestimmung je $3,0 \mathrm{~m} /$ oder weniger in andere Reagenzgläser übertragen. Die Lösungen werden mit Wasser auf $4,0 \mathrm{~m} /$ verdünnt und unter Schütteln je $2,0 \mathrm{~m} /$ Nesslerreagens zugegeben.

Direkte Methode: Bei einem Stickstoffgehalt unter $10 \mu \mathrm{g}$ werden die Proben nach dem Aufschluß mit Wasser direkt auf $2,0 \mathrm{~m} /$ aufgefüllt und $6,0 \mathrm{~m} /$ Nesslerreagens zugegeben. Hierbei sollen die Proben vor direktem Sonnenlicht geschützt werden. Die Standardlösungen werden analog behandelt.

\section{Messung der Farbintensität.}

Bei der Verdünnungsmethode ist die maximale Farbintensität nach $10 \mathrm{Min}$. und bei der direkten Methode nach etwa $30 \mathrm{Min}$. erreicht. Die Messung der Farbintensität wird in Proben, die unter $20 \mu \mathrm{g}$ Stickstoff im nesslerisierten Ansatz enthalten, bei $420 \mathrm{~m} \mu$ und im Falle höherer Stickstoffmengen bei $500 \mathrm{~m} \mu$ vorgenommen. Die Farbe ist bei Zimmertemperatur etwa 2 Stdn. beständig. Die unbekannten Stickstoffgehalte werden mit Hilfe der nächstliegenden Extinktionswerte der Standardlösungen ermittelt.

\section{Ergebnisse und Diskussion}

In der Tabelle sind die Analysenergebnisse von Stickstoffbestimmungen in verschiedenen Substanzen und biologischem Material, die nach der beschriebenen Methode gewonnen wurden, angegeben. Die gefundenen Werte entsprechen gut den eingesetzten Stickstoffmengen und zeigen im Vergleich mit der Destillationsmethode (4) befriedigende Übereinstimmung. Mit der Methode konnte in einer. Reihe von 17 Bestimmungen an derselben Probe im Bereich von 10-100 $\mu \mathrm{g} \mathrm{N}$ eine Genauigkeit von $\pm 0,3 \mu \mathrm{g} \mathrm{N}$ und im Bereich von $500-1000 \mu \mathrm{g} N$ eine solche von $\pm 1,6 \mu \mathrm{g} \mathrm{N}$ erreicht werden.
Tab.

Stickstoff bestimmungen in verschiedenen biologischen Substanzen

\begin{tabular}{|c|c|c|c|c|c|}
\hline Substanz & $\begin{array}{c}\text { Ein- } \\
\text { waage } \\
\mu \mathrm{g}\end{array}$ & $\begin{array}{c}\text { gegeben } \\
\mu \mathrm{g}\end{array}$ & $\begin{array}{l}\text { Sticks } \\
\text { Gefunden } \\
\text { nach } \\
\text { dieser } \\
\text { Methode*) } \\
\mu \mathrm{g}\end{array}$ & $\begin{array}{c}\%- \\
\text { Theorie }\end{array}$ & $\begin{array}{c}\text { Mikro- } \\
\text { methode } \\
\text { mitDestilla- } \\
=\quad \text { tion (4) } \\
\mu \mathrm{g}\end{array}$ \\
\hline L-Lysin. $\mathrm{HCl}$ & $\begin{array}{r}65 \\
649 \\
2750 \\
3660\end{array}$ & $\begin{array}{r}9,9 \\
98,9 \\
423,0 \\
564,0\end{array}$ & $\begin{array}{r}9,8 \\
99,1 \\
421,7 \\
566,3\end{array}$ & $\begin{array}{r}99,0 \\
100,2 \\
99,7 \\
100,4\end{array}$ & $\begin{array}{r}9,4 \\
99,6 \\
422,3 \\
567,0\end{array}$ \\
\hline L-Arginin. $\mathrm{HCl}$ & $\begin{array}{r}37 \\
375 \\
3925 \\
3900\end{array}$ & $\begin{array}{r}9,7 \\
97,1 \\
1044,0 \\
1037,0\end{array}$ & $\begin{array}{r}9,5 \\
97,2 \\
1055,5 \\
1041,2\end{array}$ & $\begin{array}{r}97,8 \\
100,1 \\
101,2 \\
100,4\end{array}$ & $\begin{array}{r}9,6 \\
98,2 \\
1042,0 \\
1034,7\end{array}$ \\
\hline Hämoglobin & $\begin{array}{r}59 \\
595 \\
2910 \\
10415\end{array}$ & $\begin{array}{r}10,2 \\
101,8 \\
488,0 \\
1749,0\end{array}$ & $\begin{array}{r}10,2 \\
101,8 \\
486,1 \\
1745,5\end{array}$ & $\begin{array}{r}99,8 \\
100,0 \\
100,4 \\
100,2\end{array}$ & $\begin{array}{r}10,1 \\
102,0 \\
481,3 \\
1752,6\end{array}$ \\
\hline $\begin{array}{l}\text { Rinderserum- } \\
\text { Albumin }\end{array}$ & $\begin{array}{r}62 \\
622 \\
3450\end{array}$ & $\begin{array}{r}9,9 \\
99,4 \\
579,0\end{array}$ & $\begin{array}{r}9,8 \\
99,2 \\
579,1\end{array}$ & $\begin{array}{r}98,5 \\
99,8 \\
100,0\end{array}$ & $\begin{array}{r}9,4 \\
99,6 \\
581,3\end{array}$ \\
\hline Rattenhaut & & & 60,7 & & 59,6 \\
\hline Gewebskultur & & & 14,8 & & 14,1 \\
\hline Detroit-Zellen & & & 34,0 & & 33,8 \\
\hline
\end{tabular}

*) Mittelwerte von je 3 Bestimmungen.

Die Verwendung des Aufschlußgerätes (Abb. 1) bietet gegenüber den üblichen Einrichtungen oder dem Sandbad (22) mehrere Vorteile. Durch die gute Temperaturregelung und die Möglichkeit einer schnelleren Temperaturerhöhung des Aluminiumblockes konnten die Aufschlußzeiten beträchtlich verkürzt werden. In den meisten Fällen werden die Proben schon während der Erhitzung auf $320^{\circ}$, das ist nach etwa 20-30 Min., klài und farblos. Die anschließende $30 \mathrm{Min}$. dauernde Nacherhitzung dient lediglich dazu, die von Lake (27) angenommenen schwer zersetżbaren Pyridinium-Verbindungen, die im Laufe des Aufschlusses gebildet werden, in Ammoniumsalze umzuwandeln. Dieses Verfahren ist in apparativer Hinsicht im Vergleich zu den in der Einleitung erwähnten Methoden recht einfach. Alle Arbeitsvorgänge werden in Reagenzgläsern und bei der direkten Methode ohne jegliches Umgießen der Probenlösungen vorgenommen; Pipettierfehler werden auf diese Weise weitgehend unterbunden. Da sowohl feste als auch flüssige Proben verarbeitet werden können, bieten sich der Methode sehr breite Anwendungsmöglichkeiten an.

Die Anzahl der gleichzeitig ausführbaren Analysen ist lediglich von der Zahl der Bohrungen im Aufschlußgerät abhängig. Mit dem in der Abbildung 1 wiedergegebenen Aufschlußgerät (61 Bohrungen) können täglich etwa 200 Stickstoffanalysen ausgeführt werden. Wie aus der Tabelle ersichtlich ist, liefert die Methode befriedigende Ergebnisse im Bereich von $10 \mu \mathrm{g}$ bis über $1700 \mu \mathrm{g}$ Stickstoff. 
Die Aufschlußtemperatur liegt unter dem Siedepunkt des verwendeten Aufschlußreagenzes. Das verhindert etwaige Verluste, die durch Spritzen oder Schäumen leicht entstehen können. Die Aufschlußzeit wird dadurch jedoch etwas verlängert. Eine Erhöhung der Aufschlußtemperatur auf die optimale Temperatur von $370^{\circ}(4,27)$ ist bei der angegebenen Aufschlußlösung wegen der begrenzten Löslichkeit des Kaliumsulfates nicht möglich. Um diese Temperatur zu erreichen, müßte man den Proben entsprechende Mengen festes Kaliumsulfat zugeben. Dies würde einen zusätzlichen Arbeitsgang in die Methode hineinbringen.

Der Mittelwert der $1 \mu \mathrm{g}$ Stickstoff entsprechenden Extinktion ist bei $420 \mathrm{~m} \mu$ im Bereich von $2-20 \mu \mathrm{g}$ und bei $500 \mathrm{~m} \mu$ im Bereich von 10-60 $\mu \mathrm{g}$ konstant. Oberhalb dieser Bereiche werden die mittleren Extinktionswerte immer niedriger. Dies hat systematisch zu niedrige Resultate zur Folge. Aus diesem Grunde soll aus Proben mit sehr schwankenden Stickstoffgehalten bei der Verdünnungsmethode eine Reihe von verschiedenen Volumanteilen der Grundlösung zur Nesslerisierung entnommen werden. Bei zu hohen Stickstoffkonzentrationen tritt nach kurzer Zeit eine Ausflockung des mit dem Nesslerreagens entwickelten Farbstoffes ein, die eine quantitative Auswertung der Farbintensität unmöglich macht.

Die Konzentration der überschüssigen Natronlauge in den Proben nach Nesslerisierung ist unter Einhaltung der angegebenen Vorschrift bei der Verdünnungs- methode $0,68 n$ oder höher und bei der direkten Méthode etwa 1,32n. Diese Alkalität genügt um Trübungen, die bei einigen Methoden oft eintreten (25) und eine quantitative Auswertung der Proben sehr erschweren, zu beseitigen. Sogar Metallionen, welche die Farbentwicklung schon in Spurenkonzentrationen beträchtlich beeinflussen $(24,28)$, haben bei der beschriebenen Methode bis zu Konzentrationen von etwa $10^{-3} \mathrm{~m}$ im Testansatz keine Trübungen hervorgerufen. Allerdings wird in solchen Fällen eine Zugabe der entsprechenden Ionen zu Standardlösungen empfohlen, da diese auch ohne Trübung eine Veränderung der Farbintensität verursachen (28).

Die Entwicklung der Farbreaktion ist beim Nesslerreagens bekanntlich nicht sehr gut reproduzierbar (26). Aus diesem Grund ist die Verwendung einer Eichkurve, wie bei anderen kolorimetrischen Bestimmungsmethoden, nicbt zu empfehlen. Die unbekannten Stickstoffwerte müssen deshalb mit Hilfe von Standardlösungen, die mit dem Testansatz parallel laufen, ermittelt werden. - $\mathrm{Da}$ für die Stickstoffbestimmung nur ein Teil der aufgeschlossenen Probenlösung verwendet wird, kann die restliche Lösung zur Metallbestimmung herangezogen werden.

Die beschriebene Methode eignet sich recht gut zu routinemäßigen Stickstoffbestimmungen, obwohl sie als Kjeldahl-Methode bei bestimmten Substanzklassen, die im biologischen Material jedoch selten auftreten, nur einen Teil des Stickstoffs erfaßt.

\section{Literatur}

1. BeEt, A. E., Nature (London) 175, 513 (1955). - 2. BRADStreet, R. B., Analytic. Chem. 26, 185 (1954). - 3. McCutchan, Pr. und W.F. Rorh, Analytic. Chem. 24, 369 (1962). - 4. DermelJ, M. und L. Strauch, Mikrochim. Acta (Wien) 1957, 96. - 5. Eder, K., Mikrochim. Acta (Wien) 1957, 227. - 6. Heyns, K. und G. Anders, Hoppe-Seyler's Z. physiol. Chem. 287, 109 (1951). - 7. KIRSten, W., Analytic. Chem. 24, 1078 (1952). - 8. Miller, L. und J. A. Houghton, J. biol. Chemistry 159, 373 (1945). - 9. Dumazert, Chr̈. und Y. MarCELET, Bull. Soc. Chim. biol. 20, 201 (1938). - 10. Conway, E. J., Microdiffusion analysis and volumetric error, 4. Aufl., Crosby Lockwood \& Son LTD, London (1957). - 11. ConwaY, E. J. und E. O'MALlex, Biochem. J. 36, 655 (1942). Vgl. Ref. 10, S. 12 und 68. - 12. Frscher, F. G. und H. BohN, Hoppe-Seyler's Z. physiol. Chem. 302, 278 (1955). - 13. Nathan, D. G. und K. S. Warren, Arch. Biochem. Biophysicss 81, 377 (1959). - 14. ExLEY, D., Biochem. J. 63, 496 (1956). Vgl. Russeli, J. A., J. biol. Chemistry 156, 457 (1944) und Rilley, J. P., Analytica chim. Acta (Amsterdam) 9; 575 (1953). - 15. Borssonnas, R. A. und C. H. Haselbach, Helv. chim. Acta 36, 576 (1953). 16. JACOBS, $S$., The determination of total nitrogen in small quantities of serum by the indanetrione hydrate method. Protides of the biological fluids, Proc. of the 10th Coll., Bruges 1962, S. 332, Elsevier, Amsterdam (1963). - 17. Vgl. Nathan, D. G. und F. L. Rodkey, J. Lab. clin. Med. 49, 779 (1957). - 18. Anderson, A. B., Biochem. J. 52, (1952). - 19. Boell, E. J. und S. C. Shen, Exp. Cell Res. 7, 147 (1954). - 20. Shaffer, F. L. und J. C. SPRECher, Analytic. Chem. 29, 437 (1957). 21. Vgl. Thompson, J. C. und G. R. Morrison, Analytic. Chem. 23, 1153 (1951) und Yuen, S. H. und A. G. Pollard, J. Sci. Food Agric. 3, 441 (1952). - 22. LANG, C. A., Analytic. Chem. 30, 1692 (1958). - 23. Williams, P. C., Analyst 89, 276 (1964). - 24. Minari, O. und D. B. Zilversmit, Analyt. Biochem. (New York) 6, 320 (1963). - 25. CLEGHORN, R. A. und L. JeNDRASSIK, Biochem. Z. 274, 189 (1934). - 26. Koch, F. C. und T. L. McMeekin, J. Amer. chem. Soc. 46, 2066 (1924). Vgl. Hawk, P. B., B. L. Oser und W. H. Summerson, Practical physiological chemistry, 13. Aufl., S. 1329, McGraw-Hill Book Comp., New York (1954). - 27. LAKE, G. R., Analytic. Chem. 24, 1806 (1952). - 28. Miller, G. L. und E. E. Mrller, Analytic. Chem. 20, 481 (1948).
Dr. Ludvik Strauch Max-Planck-Institut für Eiweiß- und Lederforschung 8 München 15, Schillerstraße 46 